\title{
La lymphogranulomatose vénérienne au Québec : recrudescence chez les hommes ayant des relations sexuelles avec d'autres hommes
}

\author{
CA Boutin ${ }^{1}$, S Venne ${ }^{2}$, M Fiset ${ }^{2}$, C Fortin ${ }^{1,3}$, D Murphy ${ }^{3}$, A Severini ${ }^{4}$, C Martineau ${ }^{1,3}$, J Longtin $^{3}$, \\ AC Labbé1,3*
}

\section{Résumé}

Contexte : La lymphogranulomatose vénérienne (LGV) est une infection transmise sexuellement (ITS) causée par les génotypes $L_{1}, L_{2}$ et $L_{3}$ de Chlamydia trachomatis. La LGV est associée à une morbidité considérable et à un risque accru de transmission du $\mathrm{VIH}$. Bien que moins de deux cas par an aient été rapportés au Québec avant 2005, la LGV a émergé en 2005 à 2006 avec 69 cas, suivie d'une période de faible incidence (de 2007 à 2012) et d'une recrudescence depuis 2013.

Objectif : Décrire l'incidence de la LGV au Québec et les caractéristiques de la population touchée, y compris les données démographiques et les facteurs de risque, les manifestations cliniques, les analyses de laboratoire, les traitements et les taux de réinfection.

Méthodologie : Les données descriptives relatives aux déclarations de LGV, colligées dans le fichier des maladies à déclaration obligatoire (MADO) de 2005 à 2015, ont été extraites du portail de l'infocentre de I'Institut national de santé publique du Québec (INSPO). Les questionnaires ont été obtenus dans le cadre de la vigie rehaussée et transmis de façon anonyme au ministère de la Santé du Québec. Une analyse approfondie a été effectuée sur les cas de 2013 à 2016.

Résultats : Il y a eu 338 cas de LGV au Québec au cours de cette période de quatre ans. Tous les cas étaient de sexe masculin, à l'exclusion d'un cas transsexuel. L'âge moyen était de 41 ans. La plupart vivaient à Montréal (81\%) et étaient des hommes ayant des relations sexuelles avec d'autres hommes (HARSAH; $99 \%$ ). La majorité (83\%) ont signalé quatre partenaires sexuels ou plus au cours de la dernière année, rencontrés en grande partie sur Internet (77\%) et dans les saunas (73\%). La fréquence des rapports sexuels avec des partenaires hors Québec a diminué en 2013 à 2016 (27\%) par rapport à 2005 à 2012 (38\%). Les antécédents d'ITS étaient fréquents: $83 \%$ étaient infectés par le $\mathrm{VIH}, 81 \%$ et $78 \%$ ont déclaré des antécédents de syphilis et gonorrhée, respectivement. La consommation de drogues à usage récréatif était fréquente (57\%) et à la hausse, atteignant $71 \%$ en 2016. La plupart des cas étaient symptomatiques, une proportion qui a diminué en 2016 (68\%) par rapport à la période de 2013 à 2015 (82 \%; $p=0,006)$. Les manifestations cliniques incluent la rectite (86 \%), la lymphadénopathie (13\%) et l'ulcère ou la papule (12\%). Des réinfections se sont produites chez 35 personnes (10\%), principalement dans les deux années suivant la première infection.

Conclusion : La réémergence de la LGV au Québec est décrite au sein d'une sous-population urbaine composée presque exclusivement d'HARSAH ayant des antécédents d'ITS, un nombre élevé de partenaires et consommant des drogues.

\author{
Affiliations \\ 1 Département de microbiologie, \\ infectiologie et immunologie, \\ Université de Montréal, Montréal, \\ QC \\ ${ }^{2}$ Ministère de la santé et des \\ services sociaux du Québec, \\ Montréal, QC \\ ${ }^{3}$ Institut national de santé \\ publique du Québec, Montréal, \\ QC \\ ${ }^{4}$ Laboratoire national de \\ microbiologie, Agence de la santé \\ publique du Canada, Winnipeg \\ (Manitoba)
}

*Correspondance: ac.labbe@ umontreal.ca

Citation proposée : Boutin CA, Venne S, Fiset M, Fortin C, Murphy D, Severini A, Martineau C, Longtin J, Labbé AC. La lymphogranulomatose vénérienne au Québec : recrudescence chez les hommes ayant des relations sexuelles avec d'autres hommes. Relevé des maladies transmissibles au Canada. 2018;44(2):61-8. https://doi.org/10.14745/ccdr.v44i02a04f

\section{Introduction}

La lymphogranulomatose vénérienne (LGV) est une infection transmise sexuellement (ITS) causée par les génotypes $L_{1}, L_{2}$ et $L_{3}\left(L_{1-3}\right)$ de Chlamydia trachomatis. Elle est associée entre autres à des fistules et sténoses anogénitales et à l'obstruction lymphatique (1) ainsi qu'à l'augmentation du risque de transmission du VIH (1-3). La LGV a rarement été observée dans les pays industrialisés jusqu'au début des années 2000. Depuis, elle a été décrite principalement en milieu urbain, chez 
les hommes ayant des relations sexuelles avec d'autres hommes (HARSAH). Les récentes éclosions sont survenues en Belgique, en France, aux Pays-Bas, au Royaume-Uni et aux États-Unis (2). Au cours de la dernière décennie, la LGV a aussi fait son apparition au Canada avec des flambées sporadiques principalement dans les grands centres urbains (3-5).

Au Québec, moins de deux cas par an étaient déclarés avant qu'une augmentation significative soit notée en 2005 et en 2006, avec un total de 69 cas. Une période de faible incidence a suivi, avec une moyenne de 9 cas par an entre 2007 et 2012. Puis en 2013, une épidémie a pris naissance à Montréal.

Le présentation classique de la LGV est un syndrome inguino-génital, incluant un ulcère ou une papule au site d'inoculation et une lymphadénopathie inguinale ou fémorale, fréquemment unilatérale (1). Dorénavant, plusieurs jeunes patients se présentent avec des symptômes de rectite ou de proctocolite, imitant la première manifestation de la maladie intestinale inflammatoire (2). Des études de prévalence récentes ont aussi démontré une plus large proportion de cas asymptomatiques, représentant jusqu'à $25 \%$ à $27 \%$ des cas chez les HARSAH $(6,7)$.

En 2014, l'Agence de la santé publique du Canada (ASPC) a révisé ses recommandations pour encourager le génotypage de la LGV sur les spécimens de C. trachomatis positifs provenant d'HARSAH asymptomatiques avec des facteurs de risque, quel que soit le spécimen clinique; actualisées en 2017 pour se concentrer sur les spécimens rectaux seulement (3). À la suite de la publication de conseils d'experts de l'Institut national de santé publique du Québec (INSPQ) (8), le génotypage systématique de tous les spécimens rectaux positifs pour la $C$. trachomatis (quelle que soit la symptomatologie) a été mis en œuvre en juin 2016 (la valeur de cette stratégie sera évaluée après deux ans). L'INSPO fournit également des lignes directrices sur le traitement des cas et des partenaires (8).

Compte tenu de l'épidémie croissante, la vigie rehaussée des cas de LGV a été poursuivie au Québec, visant la mise en œuvre d'interventions de santé publique ciblées.

L'objectif de cet article est de décrire l'épidémie de LGV au Québec de 2013 à 2016 et de présenter des données épidémiologiques, y compris les données démographiques, les facteurs de risque, les manifestations cliniques, les analyses de laboratoire, le traitement et la réinfection.

\section{Méthodologie}

Jusqu'en 2005, la LGV faisait partie du programme de surveillance des maladies à déclaration obligatoire (MADO) rapportées aux autorités de santé publique. En 2005, I'ASPC (9) et le ministère de la Santé du Québec ont lancé une vigie rehaussée selon laquelle, lorsqu'un cas est déclaré, les informations épidémiologiques et cliniques importantes sont recueillies par les infirmières de la santé publique, qui contactent le médecin traitant ou le patient directement. Les infirmières utilisent un questionnaire épidémiologique pour recueillir des informations sur les caractéristiques démographiques des patients (âge, sexe, lieu de résidence), les facteurs de risque (nombre, sexe et contexte de rencontre des partenaires; historique d'infections transmissibles sexuellement et par le sang [ITSS]; consommation de drogues), les manifestations cliniques, les analyses de laboratoire réalisés et les traitements reçus. Afin de diminuer les données manquantes, les questionnaires ont été revus durant la période de surveillance et les équipes médicales contactées lorsque nécessaire.

Pour ce rapport de surveillance, les données descriptives pour tous les cas de LGV au Québec de 2013 à 2016 ont été recueillies à partir de la base de données des MADO par le biais du portail de l'infocentre de I'INSPQ. Les questionnaires de la vigie rehaussée ont été transmis de façon anonyme au ministère provincial de la Santé pour compilation et analyse. Les définitions de surveillance ont évolué au fil du temps. En 2013 et en 2014, les cas confirmés incluaient:

- Les patients présentant une rectite, une lymphadénopathie inguinale/fémorale ou un contact avec un cas confirmé de LGV, et

- L'isolement de C. trachomatis ou test d'amplification des acides nucléiques (TAAN), à partir d'un échantillon clinique approprié et un génotype $L_{1-3}$ documenté par le séquençage d'ADN.

En 2015, le génotypage est devenu à lui seul suffisant pour considérer un cas confirmé, et la manifestation clinique ou le contact avec un cas n'était plus requis. Les cas probables incluaient des patients qui présentaient une rectite, une lymphadénopathie inguinale/fémorale ou ayant eu un contact avec un cas confirmé de LGV, et ayant un spécimen clinique approprié positif pour C. trachomatis (10).

Jusqu'en février 2016, tous les génotypages étaient effectués au Laboratoire national de microbiologie (LNM), à Winnipeg, au Manitoba. En mars 2016, un test d'amplification en chaîne de la polymérase (PCR) multiplexe distinguant les infections C. trachomatis LGV des non-LGV (11) est devenu disponible au Laboratoire de santé publique du Québec (LSPQ), à Sainte-Anne-de-Bellevue. Depuis, seuls les échantillons LGV positifs sont envoyés au LNM pour l'identification du génotype par séquençage de I'ADN.

Tous les cas déclarés de 2013-2016 ont été étudiés afin

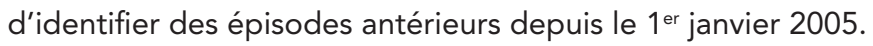
Une réinfection était définie comme une seconde infection chez le même individu, se produisant au moins 90 jours après le premier épisode (12).

Les données ont été analysées pour l'ensemble des 338 cas (ou pour les cas pour lesquels l'information était disponible pour une variable spécifique) déclarés entre 2013 et 2016, à l'exclusion du cas d'un transsexuel (un homme à une femme). Les données ont été analysées avec les logiciels Epi Info version 7.2.0.1 et Stata 64 (version 10.1). Les proportions ont été comparées en utilisant le test $x 2$. Le portrait épidémiologique présenté ici met l'accent sur l'éclosion de 2013 à 2016 au Québec, incluant 
certaines comparaisons avec les années précédentes (c.-à-d. 2005 à 2012) lorsque pertinent.

\section{Résultats}

Figure 1 : La lymphogranulomatose vénérienne chez les hommes : taux annuel ajusté par 100000 personnes-année à Montréal, hors Montréal et auQuébec, Québec, 2005 à 2016

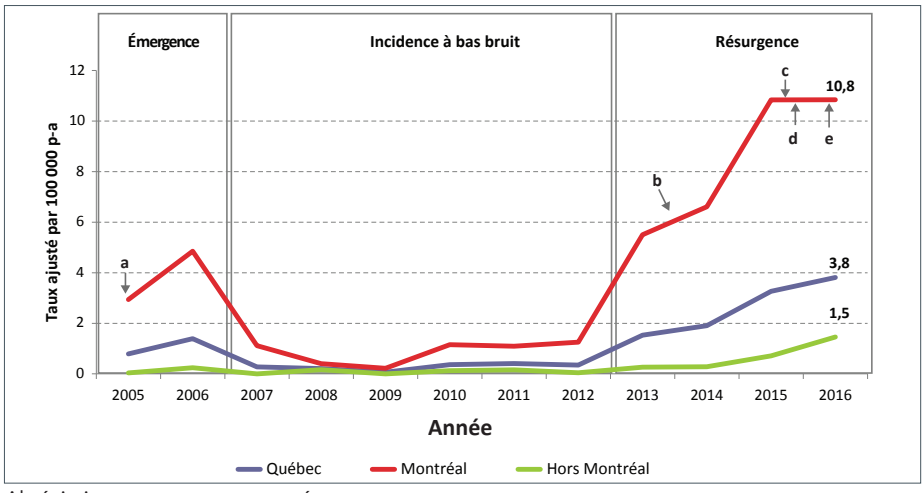

Abréviation: P-a, personne-année

2005 : Début de la vigie rehaussée de la LGV au Québec

${ }^{b}$ Septembre 2014 : Inclusion des cas asymptomatiques

c Février 2016: Transfert de l'analyse de génotypage au Québec

Juin 2016 : Génotypage systématique des spécimens $C$. trachomatis

e Novembre 2016 : Retrait de la détection sérologique

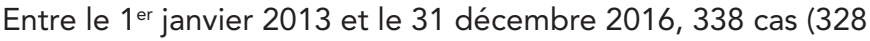
confirmés et 10 probables) ont été déclarés : 49 en 2013, 61 en 2014, 105 en 2015 et 123 en 2016 (figure 1).

\section{Données démographiques et facteurs de risque}

La majorité des cas se retrouvait dans le groupe d'âge de 25 à 54 ans (83\%) et à Montréal (81\%) (tableau 1). La plupart des patients étaient des HARSAH (99\%), dont $3 \%(n=7)$ avaient également des partenaires féminins. La moitié des patients ont déclaré plus de 10 partenaires sexuels au cours des 12 derniers mois $(101 / 205 ; 49 \%)$. Presque tous (96\%) avaient un nouveau partenaire et $84 \%$ des partenaires sexuels anonymes. La proportion de cas ayant eu plus de 20 partenaires sexuels dans la dernière année était plus élevée à Montréal que dans le reste de

Tableau 1 : Facteurs de risque associés aux cas de lymphogranulomatose vénérienne au Quebec, 2013 à 2016

\begin{tabular}{|c|c|c|}
\hline \multirow{2}{*}{ Facteurs } & \multicolumn{2}{|c|}{2013 à 2016} \\
\hline & $\mathrm{n}$ & $\%$ \\
\hline \multicolumn{3}{|l|}{ Âge $(n=338)$} \\
\hline De 15 à 24 ans & 23 & 7 \\
\hline De 25 à 34 ans & 98 & 29 \\
\hline De 35 à 44 ans & 92 & 27 \\
\hline De 45 à 54 & 83 & 25 \\
\hline 55 et plus ans & 42 & 12 \\
\hline \multicolumn{3}{|l|}{ Résidence ( $n=338$ ) } \\
\hline Montréal & 273 & 81 \\
\hline Hors Montréal & 65 & 19 \\
\hline
\end{tabular}

Tableau 1 : Facteurs de risque associés aux cas de lymphogranulomatose vénérienne au Quebec, 2013 à $2016^{\text {aa }}$ (suite)

\begin{tabular}{|c|c|c|}
\hline \multirow{2}{*}{ Facteurs } & \multicolumn{2}{|c|}{2013 à 2016} \\
\hline & $\mathbf{n}$ & $\%$ \\
\hline \multicolumn{3}{|l|}{ Sexe des partenaires ( $n=287$ ) (suite) } \\
\hline Hommes seulement & 279 & 97 \\
\hline Hommes et femmes & 7 & 2 \\
\hline Femmes seulement & 1 & 0.3 \\
\hline \multicolumn{3}{|l|}{ Histore d'ITSS ${ }^{b}$} \\
\hline Au moins une & $286 / 295$ & 97 \\
\hline $\mathrm{VIH}$ & $210 / 252$ & 83 \\
\hline Syphilis & $183 / 226$ & 81 \\
\hline Gonorrhée & $165 / 211$ & 78 \\
\hline VHB & $11 / 102$ & 11 \\
\hline $\mathrm{VHC}$ & $11 / 106$ & 10 \\
\hline \multicolumn{3}{|c|}{ Durant les 12 derniers mois : Nombre de partenaires $(n=205)$} \\
\hline 1 à 3 & 34 & 17 \\
\hline 4 à 10 & 70 & 34 \\
\hline 11 à 20 & 55 & 27 \\
\hline 20 et plus & 46 & 22 \\
\hline \multicolumn{3}{|l|}{ Contexte de rencontre ${ }^{b}$} \\
\hline Nouveau partenaire & $114 / 119$ & 96 \\
\hline Partenaires anonymes & $82 / 98$ & 84 \\
\hline Client de travailleur du sexe & $8 / 191$ & 4 \\
\hline Travailleu du sexe & $10 / 196$ & 5 \\
\hline Sauna & $136 / 186$ & 73 \\
\hline Internet/Applications & $136 / 177$ & 77 \\
\hline Club/bar & $40 / 115$ & 35 \\
\hline \multicolumn{3}{|l|}{ Drogue $(n=241)$} \\
\hline Au moins une & 138 & 57 \\
\hline Crystal meth & 50 & 21 \\
\hline Hash-pot & 48 & 20 \\
\hline Ecstasy & 43 & 18 \\
\hline Cocaïne & 28 & 12 \\
\hline Poppers & 27 & 11 \\
\hline Speed & 23 & 10 \\
\hline \multicolumn{3}{|l|}{ Droque ( $n=241$ ) (suite) } \\
\hline GHB & 12 & 5 \\
\hline Kétamine & 10 & 4 \\
\hline Crack & 5 & 2 \\
\hline Héroïne & 1 & 0 \\
\hline DIV & 10 & 4 \\
\hline Non rapportée & 13 & 5 \\
\hline \multicolumn{3}{|c|}{ Relationi avec un résident hors province $(n=208)$} \\
\hline $\begin{array}{l}\text { Au moins une } \\
\text { Abréviations : DIV, drogue intra-veineuse; } \\
\text { transmissible sexuellement; } n \text {, nombre, } V \\
\text { Patients avec ré-infections inclus plus } d^{\prime} \text { ' } \\
\text { ' La proportion pour laquelle l'information }\end{array}$ & $\begin{array}{l}\quad 56 \\
\text { te; ITSS, inf } \\
\text { :, virus de l' } \\
\text { cune des va }\end{array}$ & $\begin{array}{l}27 \\
C\end{array}$ \\
\hline
\end{tabular}

RMTC • Le 1er février 2018 • Volume 44-2

Page 63 
la province ( $27 \%$ contre $3 \% ; p<0,001$ ). Les saunas et l'Internet (y compris les applications de réseautage sur téléphones intelligents) étaient deux moyens importants pour la rencontre de partenaires. Bien que non statistiquement significatif $(p=0,07)$, moins de patients $(27 \%)$ avaient des rencontres avec des partenaires de l'extérieur de la province au cours de l'année, contrairement aux années précédentes (2005 à 2012; $n=31 / 81$; $38 \%$ ). Parmi les cas qui vivaient à l'extérieur de Montréal, 16/42 (38\%) ont eu des partenaires provenant de Montréal ou de l'extérieur de la province.

Une forte proportion de patients (210/252; $83 \%)$ étaient séropositifs pour le VIH (une augmentation considérable comparativement à la période de 2005 à 2012 : 48/82; 59 \%; $p<0,001)$. Des antécédents de syphilis (183/226; $81 \%$ ) et d'infections gonococciques (165/211; $78 \%$ ) ont également été fréquemment déclarés. Les données sur le virus de l'hépatite $B$ (VHB) et le virus de l'hépatite $\mathrm{C}(\mathrm{VHC})$ n'étaient disponibles que pour un tiers des cas, dont $11 \%$ et $10 \%$ avaient des antécédents d'infections passées ou actuelles. La proportion des cas ayant un antécédent d'au moins une ITSS était significativement plus élevée lors de la période de 2013 à 2016 (286/295; 97 \%) qu'au cours de la période de 2005 à 2012 (95/112; 85 \%; $p<0,001)$.

Les renseignements sur la consommation de drogues étaient disponibles pour 241/338 cas, dont 138 (57\%) ont déclaré avoir consommé des drogues au cours de la dernière année. Le crystal meth (21\%), le hash-pot (20\%) et l'ecstasy (18\%) ont été les drogues les plus fréquemment utilisées. Une augmentation significative de la consommation de crystal met a été observée : $8 \%(3 / 38)$ en 2013, $12 \%$ (6/49) en 2014, $25 \%$ (19/77) en 2015 et $29 \%(22 / 76)$ en 2016 (test de tendance, $p=0,008)$.

\section{Manifestations cliniques}

Les détails concernant les manifestations cliniques étaient disponibles pour 303/338 cas. La majorité des patients présentait des symptômes au moment du prélèvement pour analyse de laboratoire ( $n=237 ; 78$ \%) (tableau 2). La proportion de cas

Tableau 2 : Présentation clinique des cas de lymphogranulomatose vénérienne au Québec de 2013 à 2016

\begin{tabular}{|c|c|c|c|c|c|c|c|}
\hline \multirow[t]{2}{*}{$\begin{array}{c}\text { Présentation } \\
\text { clinique }\end{array}$} & \multicolumn{2}{|c|}{$\begin{array}{c}2013 \text { à } \\
2016 \\
\text { (Total) } \\
n=303^{a}\end{array}$} & \multicolumn{2}{|c|}{$\begin{array}{c}2013 \text { à } \\
2015 \\
n=203^{a}\end{array}$} & \multicolumn{2}{|c|}{$\begin{array}{l}2016 \\
n=100^{a}\end{array}$} & \multirow[t]{2}{*}{$\begin{array}{c}\text { Valuer } \\
p\end{array}$} \\
\hline & n & $\%$ & $n$ & $\%$ & $n$ & $\%$ & \\
\hline Asymptomatique & 69 & 23 & 37 & 18 & 32 & 32 & 0,006 \\
\hline $\begin{array}{l}\text { Non-spécifique }{ }^{b} \\
\text { seulement }\end{array}$ & 12 & 4 & 6 & 3 & 6 & 6 & NS \\
\hline $\begin{array}{l}\text { Syndrome }(s) \\
\text { spécifique(s) }\end{array}$ & 222 & 73 & 160 & 79 & 62 & 62 & 0,002 \\
\hline Rectite & 201 & 66 & 141 & 69 & 60 & 60 & NS \\
\hline Lymphadénopathie & 30 & 10 & 23 & 11 & 7 & 7 & NS \\
\hline Ulcération/papule & 29 & 10 & 25 & 12 & 4 & 4 & NS \\
\hline
\end{tabular}

Abréviations : NS, non significatif; $n$, nombre

a Informations manquantes chez 35/338 cas (12 en 2013 à 2015 et 23 en 2016)

b Les signes et symptômes non-spécifiques incluent : malaise/asthénie, écoulement urétral,

dysurie, arthralgie, fistule anale et épidydimite

'Certains patients se sont présentés avec plus d'un syndrome spécifique $(n=35)$ asymptomatiques a augmenté de $18 \%(37 / 203)$ pour la période de 2013 à 2015 à $32 \%(32 / 100)$ en $2016(p=0,01)$, et une minorité de patients présentait des symptômes non spécifiques $(12 / 235 ; 5 \%)$. Tel qu'illustré à la figure 2 , plus d'un symptôme peut se présenter au même moment. Les symptômes comprenaient la rectite (86\%), I'ulcérations ou la papule (12\%) et la lymphadénopathie (13\%). Les plaintes associées à la rectite incluaient la présence de sang dans les selles (69/201; $35 \%)$, les douleurs anales $(70 / 201,34 \%)$ et les pertes rectales (52/201, $26 \%$ ).

Figure 2 : Distribution des manifestations cliniques des cas de lymphogranulomatose vénérienne symptomatiques, Province de Québec, 2013 à 2016 $(n=234)^{\text {a }}$

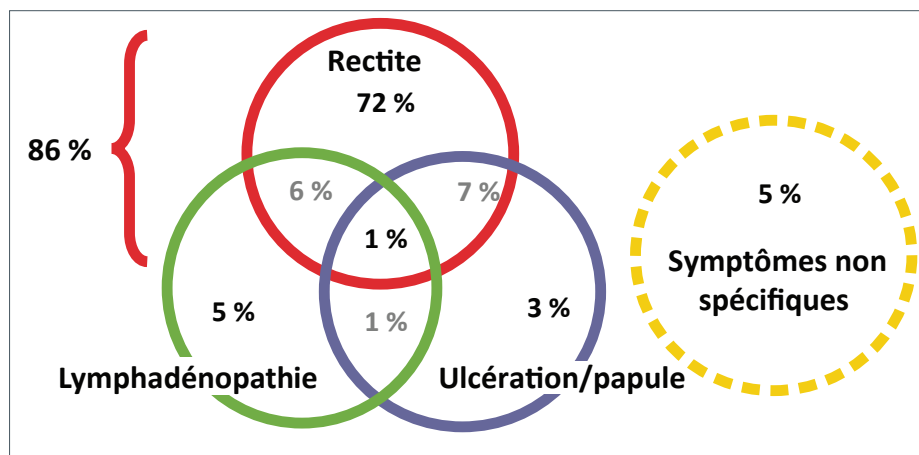

a Parmi les 338 cas, la présentation clinique étaient connue pour 303 épisodes. Un total de 69 cas était asymptomatiques

\section{Analyses de laboratoire et traitement}

Le nombre de tests de génotypage de la LGV effectués sur des spécimens de C. trachomatis positifs (hommes et femmes, tous sites d'échantillonnage, indépendamment des symptômes) au LSPQ est présenté au tableau 3. Dans l'ensemble, $13 \%$ des échantillons de C. trachomatis génotypable par PCR multiplexe étaient de génotypes $L_{1-3}$. Certains patients avaient plus d'un échantillon positif pour un seul épisode. Une importante augmentation du nombre de demandes a été notée en 2016 à la

Tableau 3 : Spécimens cliniques reçus au Laboratoire de santé publique du Québec pour le génotypage de la lymphogranulomatose vénérienne, 2013 à 2016

\begin{tabular}{|c|c|c|c|c|c|c|c|}
\hline \multirow[t]{2}{*}{ Années } & \multirow{2}{*}{$\begin{array}{c}\text { Spécimens } \\
\text { positifs } \\
\text { pour } C \text {. } \\
\text { trachomatis, } \\
\text { n }\end{array}$} & \multirow{2}{*}{$\begin{array}{c}\text { Géno- } \\
\text { typable } \\
\text { par } \\
\text { multiplex } \\
\text { PCR } \\
\text { multiplex, } \\
n\end{array}$} & \multirow{2}{*}{$\begin{array}{c}\text { Génotypes } \\
\text { non-LGV, } \\
n(\%)\end{array}$} & \multirow[t]{2}{*}{$\begin{array}{l}\text { Génotypes } \\
\text { LGV, n (\%) }\end{array}$} & \multicolumn{3}{|c|}{$\begin{array}{c}\text { Génotype } \\
\text { spécifique } \\
\text { identifié par } \\
\text { séquençage }\end{array}$} \\
\hline & & & & & $\mathrm{L}_{2 b}$ & $\mathrm{~L}_{2}$ & ND \\
\hline 2013 & 424 & 414 & 363 (88 \%) & $51(12 \%)$ & 49 & 2 & 0 \\
\hline 2014 & 540 & 534 & 475 (89 \%) & 59 (11\%) & 55 & 4 & 0 \\
\hline 2015 & 670 & 656 & 536 (82 \%) & 120 (18\%) & 107 & 4 & 9 \\
\hline 2016 & 1249 & 1199 & 1070 (89 \%) & 129 (11\%) & 124 & 0 & 5 \\
\hline Total & 2883 & 2803 & 2444 (87 \%) & 359 (13\%) & 335 & 10 & 14 \\
\hline
\end{tabular}

Abréviations : C. trachomatis, Chlamydia trachomatis; LGV, lymphogranulomatose vénérienne; $\mathrm{PCR}$, réaction en chaîne par la polymérase

anclus tous les hommes et femmes, avec spécimens de différents sites

Notes: Certains cas de lymphogranulomatose vénérienne ont été rapportés sur la base de la sérologie avant novembre 2016; certains patients ont eu plus d'un spécimen positif 


\section{Discussion}

tous les échantillons rectaux positifs pour $C$. trachomatis.

Les raisons de prélèvement du spécimen clinique était connue pour 302 des 338 cas déclarés. Elles incluaient la présence de symptômes ( $n=221 ; 73 \%$ ); le dépistage basé sur les facteurs de risque ( $n=68 ; 23 \%$ ); et le contact avec un cas confirmé de la $\operatorname{LGV}(n=12 ; 4 \%)$. Le séquençage de l'ADN a été mené auprès de $93 \%(n=314)$ des cas : 304 étaient génotypés $L_{2 b}$, alors que 10 étaient génotypés comme $L_{2}$, en raison d'une mutation d'un seul nucléotide sur la cible du gène ompA. Le séquençage rétrospectif du gène $\mathrm{pmpH}$ suggère que ces cas étaient dus à une variante du génotype $L_{2 b}$.

Le traitement prescrit était conforme aux lignes directrices (doxycycline pendant 21 jours) $(3,8)$ chez $77 \%$ des cas $(221 / 288)$, et le traitement initial approprié a été le plus souvent prescrit en 2016 comparativement à la période de 2013 à 2015 (74 \% contre $61 \% ; p=0,03$ ) (tableau 4).

Tableau 4 : Traitement des cas de lymphogranulomatose vénérienne au Québec de 2013 à 2016

\begin{tabular}{|c|c|c|c|c|c|c|c|}
\hline \multirow[t]{2}{*}{ Traitement } & \multicolumn{2}{|c|}{$\begin{array}{c}2013 \text { à } 2016 \\
\text { (Total) } \\
n=288^{a}\end{array}$} & \multicolumn{2}{|c|}{ 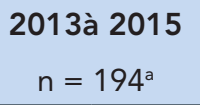 } & \multicolumn{2}{|c|}{$\begin{array}{c}2016 \\
n=94^{a}\end{array}$} & \multirow[t]{2}{*}{ Valeur $p$} \\
\hline & n & $\%$ & $n$ & $\%$ & $\mathbf{n}$ & $\%$ & \\
\hline $\begin{array}{l}\text { Traitement } \\
\text { appropriéb }^{\text {a }}\end{array}$ & 221 & 77 & 144 & 74 & 77 & 82 & NS \\
\hline $\begin{array}{l}\text { À la première } \\
\text { prescription }\end{array}$ & 189 & 66 & 119 & 61 & 70 & 74 & 0,03 \\
\hline $\begin{array}{l}\text { À la deuxième } \\
\text { prescription }\end{array}$ & 32 & 11 & 25 & 13 & 7 & 7 & NS \\
\hline $\begin{array}{l}\text { Traitment } \\
\text { inapproprié }\end{array}$ & 67 & 23 & 50 & 26 & 17 & 18 & NS \\
\hline
\end{tabular}

Abréviation : $n$, nombre; NS, non significatif

a Information manquante chez 50/338 cas (21/215 en 2013 à 2015 et 29/123 en 2016)

${ }^{6}$ Traitement approprié : Doxycycline pendant 21 jour (3)

\section{Réinfections}

Parmi les 35 personnes qui étaient des patients avec réinfection (les 338 cas sont survenus chez 303 individus), c'est-à-dire qui ont été réinfectés plus de 90 jours après un épisode, 10 (29\%) avaient eu au moins un épisode documenté avant 2013, alors que 25 avaient eu leur premier épisode au cours de la période allant de 2013 à 2016 (12). La plupart des réinfections (75 \%) se sont produites dans les deux années suivant la première infection (de 108 jours à 10,5 ans). Les patients avec réinfection étaient plus susceptibles de vivre à Montréal que ceux n'ayant eu qu'un épisode (31/35 [89\%] contre 210/268 [78 \%]; $p=0,13)$. Comparativement aux personnes n'ayant eu qu'un seul épisode, les patients avec réinfection étaient également plus susceptibles d'être infectés par le VIH (29/30 [97\%] contre 155/193 [80\%]; $p=0,03)$; d'avoir des antécédents de syphilis (27/27 [100\%] contre 132/173 [76 \%]; $p=0,004)$; et d'avoir consommé des drogues récréatives au cours des 12 derniers mois (17/22 [77\%] contre 106/194 [55\%]; $p=0,04$ ).
L'incidence de la LGV, qui évolue au sein d'un groupe d'HARSAH vivant en milieu urbain, a augmenté au Québec au cours des quatre dernières années. Cette population d'HARSAH se caractérise par la présence d'antécédents d'ITSS, en particulier le $\mathrm{VIH}$, un nombre élevé de partenaires sexuels ainsi qu'une consommation fréquente de drogues récréatives. Nos données montrent une forte prévalence de la rectite comme présentation clinique, avec très peu d'atteinte inguino-genitale classique. La majorité des cas ont été confirmés de génotype $L_{2 b}$, une variante isolée il y a déjà plusieurs décennies à San Francisco (13). Tel qu'illustré au Québec, plusieurs études de cas européennes ont dépeint une population d'HARSAH à haut risque (2). On y décrit un pourcentage important d'hommes infectés par le $\mathrm{VIH}$ ou par le virus de l'hépatite $C$, et très peu de cas chez les femmes ou les hommes hétérosexuels. Des cas asymptomatiques sont documentés, mais la grande majorité des cas présente des symptômes d'infection rectale (14-19).

Le taux de LGV demeure relativement faible par rapport à d'autres ITS courantes, y compris les infections causées par C. trachomatis non-LGV. En 2015, parmi les hommes de 15 à 75 ans au Québec, les taux d'incidence annuels de la syphilis infectieuse (17,2/100 000 personnes-année) et de l'infection gonococcique $(69,4 / 100000$ personnes-année) étaient significativement plus élevés que ceux de la LGV $(2,6 / 100000$ personnes-année) (20). Puisque l'on estime que la LGV est plus souvent symptomatique que la syphilis ou que l'infection gonococcique, il est possible de spéculer que les cas de LGV sont plus susceptibles de consulter un médecin et d'obtenir un traitement, ce qui limiterait la transmission. De plus, la LGV est pour l'instant limitée à un sous-groupe d'HARSAH, alors que les infections gonococciques et les infections à $C$. trachomatis non-LGV sont également rapportées dans la population hétérosexuelle. Toutefois, I'incidence de la LGV avant 2014 était probablement sous-estimée, compte tenu de l'exclusion des cas asymptomatiques dans les définitions nosologiques antérieures. La plus forte proportion de cas asymptomatiques en 2016 peut également s'expliquer en partie par le génotypage systématique des spécimens rectaux positifs pour $C$. trachomatis, qui a débuté en juin 2016 au Québec.

Les manifestations cliniques décrites dans ce rapport sont en accord avec la littérature récente, rapportant la rectite et la proctocolite comme les présentations les plus fréquentes $(2,21)$. Bien que le génotypage systématique de la LGV soit actuellement effectué au Québec seulement pour les spécimens rectaux, certains indices nous portent à croire que la LGV extrarectale (infections urétrale et pharyngienne) pourrait potentiellement contribuer à l'éclosion actuelle. En 2014, van Rooijen et al. (22) ont recueilli des prélèvements pharyngiens chez une population d'HARSAH, et ont constaté que $1 \%$ étaient positifs pour $C$. trachomatis; de ce nombre, $53 \%$ n'avaient pas d'infection concomitante dans la région anogénitale. Le rôle de la LGV pharyngienne, de même que celui de la LGV urétrale dans la transmission de la souche épidémique ne sont pas clairs. Toutefois, il a été démontré à travers plusieurs études de prévalence, que les infections rectales sont beaucoup plus communes (23-26). Il est donc raisonnable de douter de la rentabilité d'un dépistage extrarectal. 
Une amélioration significative des traitements initiaux adéquats a été constatée en 2016, probablement à la suite d'efforts axés sur la formation des médecins $(3,8)$. Le génotypage des spécimens rectaux positifs pour $C$. trachomatis à l'échelle de la province, mis en place en 2016, pourrait avoir contribué à l'amélioration de la gestion de cas en raccourcissant les délais diagnostiques, passant d'une moyenne de 30 jours en 2014 (9) à 12 jours en 2016 (données non présentées).

Une sous-population spécifique semble être plus à risque de réinfection, avec des taux plus élevés d'infection par le VIH et des comportements sexuels potentiellement plus risqués, comme l'indiquent d'autres rapports $(27,28)$. Dans une tentative de décrire cette sous-population, Rönn et al. (12) ont suggéré que les patients avec réinfections étaient plus susceptibles d'être infectés par le VIH et le VHC, et d'avoir une infection gonococcique concomitante, comparativement aux patients n'ayant eu qu'un seul épisode de LGV. II n'est pas clair si le sérotriage, la stratégie par laquelle les HARSAH ont des rapports sexuels non protégés avec des partenaires séroconcordants, a un rôle à jouer dans l'épidémie actuelle, comme cela est suggéré pour d'autres ITS au sein de ce groupe $(29,30)$.

La surveillance accrue de la LGV à l'échelle nationale, menée entre 2004 et 2012, a engendré 170 rapports de cas aux autorités de santé provinciales et territoriales (dont 104 cas confirmés et 66 cas probables) (5). Les cas confirmés ont été rapportés au Québec, en Ontario, en Alberta et en Colombie-Britannique; les cas probables ont également été déclarés dans ces provinces, ainsi qu'un cas en Nouvelle-Écosse (5).

\section{Points forts et limites}

Cette étude fournit des données de surveillance récentes sur la LGV au Québec avec un nombre élevé de cas. La description des facteurs de risque contribue à la compréhension de l'épidémie actuelle et de son réseau sexuel. Les limites incluent la nature descriptive et l'absence de standardisation des questionnaires, à la fois au fil du temps et entre les différentes régions administratives, ainsi que les informations manquantes. Les formulaires de collecte de données ont été révisés depuis, et les équipes médicales contactées afin de permettre une meilleure standardisation. Les données en lien avec le suivi du VIH (c.-à-d. le traitement, la charge virale, CD4, etc.) n'étaient malheureusement pas disponibles.

\section{Conclusion}

La réémergence de la LGV au Québec touche un groupe d'HARSAH ayant des antécédents d'ITS, la plupart vivant avec le $\mathrm{VIH}$, avec de multiples partenaires et une consommation importante de drogues. La transmission sporadique à l'extérieur de Montréal et les réinfections relativement fréquentes mettent en évidence le potentiel de propagation, ce qui est préoccupant considérant la morbidité associée à la LGV. La transmission chez les patients infectés par le VIH est aussi inquiétante, puisqu'elle témoigne des rapports sexuels non protégés au sein de cette population et que l'inflammation de la muqueuse rectale secondaire à la rectite est associée à un risque augmenté de transmission du VIH (1-3). La vigie rehaussée actuelle permet de suivre et de mieux décrire ce sous-groupe afin de modifier les mesures de santé publique en vue de réduire le risque de transmission de la LGV. Un outil clinique pour la LGV a été publié en octobre 2017 afin d'épauler les équipes médicales avec le dépistage, le diagnostic, le traitement et le suivi de la LGV, ainsi qu'avec l'intervention auprès des partenaires (31). Le génotypage systématique de spécimens rectaux positifs pour C. trachomatis s'est révélé utile pour reconnaître les patients asymptomatiques. Une analyse de la rentabilité de cette stratégie pourrait façonner les futures actions en matière de santé publique.

\section{Déclaration des auteurs}

C.A.B. - Rédaction - ébauche originale, examen et révision, visualisation

S.V. - Conceptualisation, méthodologie, validation, analyse officielle, enquête, rédaction - examen et révision, administration du projet

M.F. - Conceptualisation, méthodologie, logiciel, validation, analyse officielle, conservation des données, rédaction - examen et révision, administration du projet

D.M. - Méthodologie, enquêtes, collecte et conservation des données, rédaction - examen et révision

C.M. - Méthodologie, rédaction - examen et révision

A.S. - Génotypage par séquençage direct, rédaction - examen et révision

J.L. - Ressources, rédaction - examen et révision, supervision

C.F. - Rédaction - examen et révision

A.C.L. - Conceptualisation, analyse officielle, validation, rédaction - examen et révision, visualisation, supervision

\section{Conflit d'intérêt}

Aucun.

\section{Remerciements}

Les auteurs tiennent à remercier les infirmières de santé publique, les médecins collaborateurs, les directions régionales de santé publique, le ministère de la Santé et des Services sociaux du Québec, le Bureau de la Vigie sanitaire et la Direction de la lutte contre les ITSS pour la collecte et le traitement des données cliniques. Nous tenons également à remercier, pour les analyses de laboratoires, Suzanne Gibbons et Vanessa Zubach au laboratoire des exanthèmes viraux et maladies transmises sexuellement (LNM) ainsi que Martine Morin et Lyne Desautels pour la mise en œuvre de la PCR multiplexe au LSPQ. .

\section{Financement}

Cette étude épidémiologique a été soutenue par les organismes de santé publique provinciaux qui ont participé à la collecte de données et aux analyses.

\section{Références}

1. Ministère de la santé et des services sociaux du Québec. Guide québécois de dépistage de ITSS, Fiche clinique. Lymphogranulomatose vénérienne - MSSS. Québec (QC): 
MSSS; 2017. http://publications.msss.gouv.qc.ca/msss/ document-000090/

2. Stoner BP, Cohen SE. Lymphogranuloma venereum 2015: clinical presentation, diagnosis, and treatment. Clin Infect Dis 2015 Dec;61 Suppl 8:S865-73. DOI (http://dx.doi. org/10.1093/cid/civ756). PubMed (https://www.ncbi.nlm. nih.gov/entrez/query.fcgi?cmd=Retrieve\&db=PubMed\&li st_uids=26602624\&dopt=Abstract).

3. Agence de la santé publique du Canada. Lignes directrices canadiennes sur les infections transmissibles sexuellement. Ottawa (ON): ASPC; 2017. https://www.canada.ca/fr/ sante-publique/services/maladies-infectieuses/sante-sexuell e-infections-transmissibles-sexuellement/lignes-directricescanadiennes/infections-transmissibles-sexuellement.html

4. Kropp RY, Wong T; Canadian LGV Working Group. Emergence of lymphogranuloma venereum in Canada. CMAJ 2005 Jun;172(13):1674-6. DOI (http://dx.doi. org/10.1503/cmaj.050621. PubMed (https://www.ncbi.nlm. nih.gov/entrez/query.fcgi?cmd=Retrieve\&db=PubMed\&li st_uids $=15928239 \&$ dopt=Abstract).

5. Agence de la santé publique du Canada. Section 1: Rapport sur les infections transmissibles sexuellement au Canada : 2012 - Chlamydia (Chlamydia trachomatis). Ottawa (ON): ASPC; 2017. https://www.canada.ca/fr/sante-publique/ services/maladies-infectieuses/surveillance-epidemiolog y-infections-transmises-sexuellement-hep-b-c/rapport-2012/ rapport-infections-transmissibles-sexuellementcanada-2012-5.html

6. Saxon C, Hughes G, Ison C; UK LGV Case-Finding Group. Asymptomatic lymphogranuloma venereum in men who have sex with men, United Kingdom. Emerg Infect Dis 2016 Jan;22(1):112-6. DOI (http://dx.doi.org/10.3201/ EID2201.141867). PubMed (https://www.ncbi.nlm.nih. gov/entrez/query.fcgi?cmd=Retrieve\&db=PubMed\&lis t_uids=26691688\&dopt=Abstract).

7. de Vrieze NH, de Vries HJ. Lymphogranuloma venereum among men who have sex with men. An epidemiological and clinical review. Expert Rev Anti Infect Ther 2014 Jun;12(6):697-704. DOI (http://dx.doi.org/10.1586/1478 7210.2014.901169). PubMed (https://www.ncbi.nlm.nih. gov/entrez/query.fcgi?cmd=Retrieve\&db=PubMed\&lis t_uids=24655220\&dopt=Abstract).

8. Comité sur les analyses de laboratoire en lien avec les ITSS (CALI). Lymphogranulomatose vénérienne: avis sur le dépistage, la prise en charge clinique et la surveillance au Québec. Québec (QC): INSPQ; 2016. https://www.inspq. qc.ca/publications/2130

9. Agence de la santé publique du Canada. Protocole pour la surveillance accrue de LGV - Lymphagranuloma venereum (LGV) au Canada : Recommandations pour son diagnostic et son traitement Protocole de surveillance accrue à l'échelle nationale. Ottawa (ON): ASPC; 2017. http://www.phac-aspc. gc.ca/publicat/lgv/lgv-rdt2-fra.php

10. Ministère de la Santé et des Services Sociaux du Québec (MSSS). Surveillance des maladies à déclaration obligatoire au Québec - Définitions nosologiques. msss.gouv. qc.ca. 2016. http://publications.msss.gouv.qc.ca/msss/ fichiers/2016/16-268-03W.pdf
11. Chen $\mathrm{CY}$, Chi KH, Alexander S, Ison CA, Ballard RC. A real-time quadriplex PCR assay for the diagnosis of rectal lymphogranuloma venereum and non-lymphogranuloma venereum Chlamydia trachomatis infections. Sex Transm Infect 2008 Aug;84(4):273-6. DOI (http://dx.doi.org/10.1136/ sti.2007.029058). PubMed (https://www.ncbi.nlm.nih. gov/entrez/query.fcgi?cmd=Retrieve\& $\mathrm{db}=$ PubMed\&lis t_uids=18283094\&dopt=Abstract).

12. Rönn M, Hughes $G$, White $P$, Simms I, Ison C, Ward H. Characteristics of LGV repeaters: analysis of LGV surveillance data. Sex Transm Infect 2014 Jun;90(4):275-8. DOI (http:// dx.doi.org/10.1136/sextrans-2013-051386). PubMed (https:// www.ncbi.nlm.nih.gov/entrez/query.fcgi?cmd=Retrieve\&db= PubMed\&list_uids=24431182\&dopt=Abstract).

13. Spaargaren J, Schachter J, Moncada J, de Vries HJ, Fennema HS, Peña AS et al. Slow epidemic of lymphogranuloma venereum L2b strain. Emerg Infect Dis 2005 Nov;11(11):1787-8. DOI (http://dx.doi.org/10.3201/ eid1111.050821). PubMed (https://www.ncbi.nlm.nih. gov/entrez/query.fcgi?cmd=Retrieve \&db=PubMed\&lis t_uids=16318741\&dopt=Abstract).

14. Haar K, Dudareva-Vizule S, Wisplinghoff H, Wisplinghoff F, Sailer A, Jansen $\mathrm{K}$ et al. Lymphogranuloma venereum in men screened for pharyngeal and rectal infection, Germany. Emerg Infect Dis 2013 Mar;19(3):488-92. DOI (http://dx.doi. org/10.3201/eid1903.121028). PubMed (https://www.ncbi. $\mathrm{nlm}$.nih.gov/entrez/query.fcgi?cmd=Retrieve\&db=PubMed\&l ist_uids=23621949\&dopt=Abstract).

15. Spaargaren J, Fennema HS, Morré SA, de Vries HJ, Coutinho RA. New lymphogranuloma venereum Chlamydia trachomatis variant, Amsterdam. Emerg Infect Dis 2005 Jul;11(7):1090-2. PubMed (https://www.ncbi.nlm.nih. gov/entrez/query.fcgi?cmd=Retrieve $\& d b=$ PubMed\&lis t_uids $=16022786 \& d o p t=A b s t r a c t)$

16. Rönn MM, Ward $H$. The association between lymphogranuloma venereum and HIV among men who have sex with men: systematic review and meta-analysis. BMC Infect Dis 2011 Mar;11:70. DOI (http://dx.doi. org/10.1186/1471-2334-11-70). PubMed (https://www.ncbi. $\mathrm{nlm}$.nih.gov/entrez/query.fcgi?cmd=Retrieve\&db=PubMed\&l ist_uids=21418569\&dopt=Abstract).

17. de Vrieze NH, van Rooijen M, Schim van der Loeff MF, de Vries HJ. Anorectal and inguinal lymphogranuloma venereum among men who have sex with men in Amsterdam, The Netherlands: trends over time, symptomatology and concurrent infections. Sex Transm Infect 2013 Nov;89(7):548-52. DOI (http://dx.doi.org/10.1136/ sextrans-2012-050915). PubMed (https://www.ncbi.nlm. nih.gov/entrez/query.fcgi?cmd=Retrieve \&db=PubMed\&li st_uids=23427272\&dopt=Abstract).

18. Parra-Sánchez M, García-Rey S, Pueyo Rodríguez I, Viciana Fernández P, Torres Sánchez MJ, Palomares Folía JC. Clinical and epidemiological characterisation of lymphogranuloma venereum in southwest Spain, 2013-2015. Sex Transm Infect 2016 Dec;92(8):629-31. DOI (http://dx.doi.org/10.1136/ sextrans-2015-052453). PubMed (https://www.ncbi.nlm. nih.gov/entrez/query.fcgi?cmd=Retrieve\&db=PubMed\&li st_uids=27288416\&dopt=Abstract). 
19. Childs T, Simms I, Alexander S, Eastick K, Hughes G, Field N. Rapid increase in lymphogranuloma venereum in men who have sex with men, United Kingdom, 2003 to September 2015. Euro Surveill 2015;20(48):30076. DOI (http://dx.doi. org/10.2807/1560-7917.ES.2015.20.48.30076). PubMed (https://www.ncbi.nlm.nih.gov/entrez/query.fcgi?cmd=Retrie ve\&db=PubMed\&list_uids=26675210\&dopt=Abstract).

20. Blouin K, Venne S, Lambert G. Portrait des infections transmissibles sexuellement et par le sang (ITSS) au Québec: année 2015 (et projections 2016). Québec (QC): INSPQ; 2017. https://www.inspq.qc.ca/publications/2201

21. de Vrieze NH, Versteeg B, Bruisten SM, van Rooijen MS, van der Helm JJ, de Vries HJ. Low prevalence of urethral lymphogranuloma venereum infections among men who have sex with men: a prospective observational study, sexually transmitted infection clinic in Amsterdam, the Netherlands. Sex Transm Dis 2017 Sep;44(9):547-50. DOI (http://dx.doi.org/10.1097/ OLQ.0000000000000657). PubMed (https://www.ncbi.nlm. nih.gov/entrez/query.fcgi?cmd=Retrieve \&db=PubMed\&li st_uids=28809772\&dopt=Abstract).

22. van Rooijen MS, van der Loeff MF, Morré SA, van Dam AP, Speksnijder AG, de Vries HJ. Spontaneous pharyngeal Chlamydia trachomatis RNA clearance. A cross-sectional study followed by a cohort study of untreated STI clinic patients in Amsterdam, The Netherlands. Sex Transm Infect 2015 May;91(3):157-64. DOI (http://dx.doi.org/10.1136/ sextrans-2014-051633). PubMed (https://www.ncbi.nlm. nih.gov/entrez/query.fcgi?cmd=Retrieve $\& d b=$ PubMed $\&$ li st_uids=25237127\&dopt=Abstract).

23. Annan NT, Sullivan AK, Nori A, Naydenova P, Alexander S, McKenna A et al. Rectal chlamydia--a reservoir of undiagnosed infection in men who have sex with men. Sex Transm Infect 2009 Jun;85(3):176-9. DOI (http://dx.doi. org/10.1136/sti.2008.031773). PubMed (https://www.ncbi. $\mathrm{nlm}$.nih.gov/entrez/query.fcgi?cmd=Retrieve\&db=PubMed\&l ist_uids=19176570\&dopt=Abstract).

24. Rodríguez-Domínguez $M$, Puerta $T$, Menéndez $B$, González-Alba JM, Rodríguez C, Hellín T et al. Clinical and epidemiological characterization of a lymphogranuloma venereum outbreak in Madrid, Spain: co-circulation of two variants. Clin Microbiol Infect 2014 Mar;20(3):219-25. DOI (http://dx.doi.org/10.1111/14690691.12256). PubMed (https://www.ncbi.nlm.nih.gov/ entrez/query.fcgi?cmd=Retrieve \&db=PubMed\&list_ uids=23730727\&dopt=Abstract).

25. Ward H, Alexander S, Carder C, Dean G, French P, Ivens $D$ et al. The prevalence of lymphogranuloma venereum infection in men who have sex with men: results of a multicentre case finding study. Sex Transm Infect 2009 Jun;85(3):173-5. DOI (http://dx.doi.org/10.1136/ sti.2008.035311). PubMed (https://www.ncbi.nlm.nih. gov/entrez/query.fcgi?cmd=Retrieve\& $\mathrm{db}=$ PubMed\&lis t_uids=19221105\&dopt=Abstract).

26. de Vrieze NH, van Rooijen M, Speksnijder AG, de Vries HJ. Urethral lymphogranuloma venereum infections in men with anorectal lymphogranuloma venereum and their partners: the missing link in the current epidemic? Sex Transm Dis 2013 Aug;40(8):607-8. DOI (http://dx.doi.org/10.1097/01. OLQ.0000431359.26583.13). PubMed (https://www.ncbi. $\mathrm{nlm}$.nih.gov/entrez/query.fcgi?cmd=Retrieve \&db=PubMed\&l ist_uids=23859905\&dopt=Abstract).

27. Macdonald N, Sullivan AK, French P, White JA, Dean G, Smith A et al. Risk factors for rectal lymphogranuloma venereum in gay men: results of a multicentre case-control study in the U.K. Sex Transm Infect 2014 Jun;90(4):262-8. DOI (http://dx.doi.org/10.1136/ sextrans-2013-051404). PubMed (https://www.ncbi.nlm. nih.gov/entrez/query.fcgi?cmd=Retrieve \&db=PubMed\&li st_uids=24493859\&dopt=Abstract).

28. de Vries HJ, van der Bij AK, Fennema JS, Smit C, de Wolf $\mathrm{F}$, Prins $\mathrm{M}$ et al. Lymphogranuloma venereum proctitis in men who have sex with men is associated with anal enema use and high-risk behavior. Sex Transm Dis 2008 Feb;35(2):203-8. DOI (http://dx.doi.org/10.1097/ OLQ.0b013e31815abb08). PubMed (https://www.ncbi.nlm. nih.gov/entrez/query.fcgi?cmd=Retrieve \&db=PubMed\&li st_uids=18091565\&dopt=Abstract).

29. Marcus U, Schmidt AJ, Hamouda O. HIV serosorting among HIV-positive men who have sex with men is associated with increased self-reported incidence of bacterial sexually transmissible infections. Sex Health 2011 Jun;8(2):184-93. DOI (http://dx.doi.org/10.1071/SH10053). PubMed (https:// www.ncbi.nlm.nih.gov/entrez/query.fcgi?cmd=Retrieve\&db= PubMed\&list_uids=21592432\&dopt=Abstract).

30. Jin F, Prestage GP, Templeton DJ, Poynten IM, Donovan B, Zablotska I et al. The impact of HIV seroadaptive behaviors on sexually transmissible infections in HIV-negative homosexual men in Sydney, Australia. Sex Transm Dis 2012 Mar;39(3):191-4. DOI (http://dx.doi.org/10.1097/ OLQ.0b013e3182401a2f). PubMed (https://www.ncbi.nlm. nih.gov/entrez/query.fcgi?cmd=Retrieve $\& d b=$ PubMed $\&$ li st_uids=22337105\&dopt=Abstract).

31. Ministère de la santé et des services sociaux du Québec (MSSS). Recrudescence de la lymphogranulomatose vénérienne au Québec: détection et traitement. Québec (QC): Gouvernement du Québec; 2017. http://publications. msss.gouv.qc.ca/msss/document-001956/ 